Témoigner Témoigner. Entre histoire et mémoire

Getuigen Revue pluridisciplinaire de la Fondation Auschwitz

118 | 2014

Au nom des victimes. Dictature et terreur d'État en Argentine, Chili et Uruguay

\title{
Laboratoire mémoriel Belgique-België
}

Dans le prochain numéro

Memory laboratory Belgium-Belgique

Herinneringslabo Belgie-Belgique

\section{OpenEdition}

Journals

Édition électronique

URL : http://journals.openedition.org/temoigner/1078

DOI : 10.4000/temoigner.1078

ISSN : 2506-6390

Éditeur :

Éditions du Centre d'études et de documentation Mémoire d'Auschwitz, Éditions Kimé

Édition imprimée

Date de publication : 1 octobre 2014

Pagination : 226

ISBN : 978-2-84174-674-3

ISSN : 2031-4183

Référence électronique

"Laboratoire mémoriel Belgique-België », Témoigner. Entre histoire et mémoire [En ligne], 118 | 2014, mis en ligne le 01 octobre 2015, consulté le 23 octobre 2020. URL : http://journals.openedition.org/ temoigner/1078; DOI : https://doi.org/10.4000/temoigner.1078 


\section{Rwanda}

\section{LES ÉGLISES-CHARNIERS DU GÉNOCIDE : LE CAS DE KIBEHO}

$\diamond$ Par Rémi Korm
EHESS (École des EHESS (École des
Hautes Etudes en Sciences Sociales)

II effectue des
recherches universitir

recherches universit
sur la mémoire du

génocide des Tutsi

au Rwanda où il se

rend régulièrement.

II développe en

parallèle des projets de

transmission publique

leassociation lbuka à

Lyon.

$\Delta$ Cette chronique

régulière vise à

présenter la façon don

se construit le souvenir

du génocide des Tutsi

1994. Elle prendra

1994. Elle prendra en

du modèle mémoriel

rwandais, mais aussi

les nombreux liens

tissés avec des

institutions travaillant

sur la mémoire de la

Shoah, sur le génocid

des Arméniens ou

encore le génocide
cambodgien.

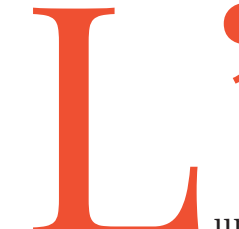

9

une des spécificités du génocide des Tutsi au Rwanda est toute la population, et surtor, davant porticipation d'une partie d'e avec lle. Cette dimension populaire du génocide a eu lieu sur tout le territoire. Des centaines de milliers de Tutsi ont ain. été assassinés par petits groupes sur leurs collines par leurs vorins. Mais leurs cours du génocide, des massars collecis de genande des massares coltectifs degrandee été organisés.

Apres lattentat du 6 avril 199 ayant coûté la vie au président Juvése réfugièrent, parfois encouragés par les autorités, au sein de lieux publics. les autorites, au sein de lieux publics. Ce fut principalement le cas dans les les lieux de culte et mais asi églises catholiques. Avant 1994, cellesci avaient servi de refuge aux civils tutsi menacés. Mais pour la première fois, au cours des trois mois du génocide elles ont été transforAut leu de massacre.

Anterogations sur les éments ayant rendu possible un te

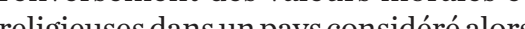
clige distique subconn question du dever de cos guestion du devenir de ces sites. Condeséglises latili

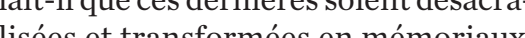

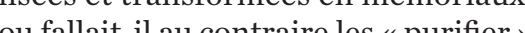
ou fallait-il au contraire les upurifier et reprendre le culte.

Afnn de statuer sur le devenir de ces lieux, une commission mixte fut instithe $1996^{2}$. Cette derière fut wané en $1906^{2}$. Cette dernière fut

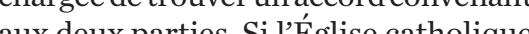
rwa deux parties. Si lighise catholique certain était conciliante et prête à 


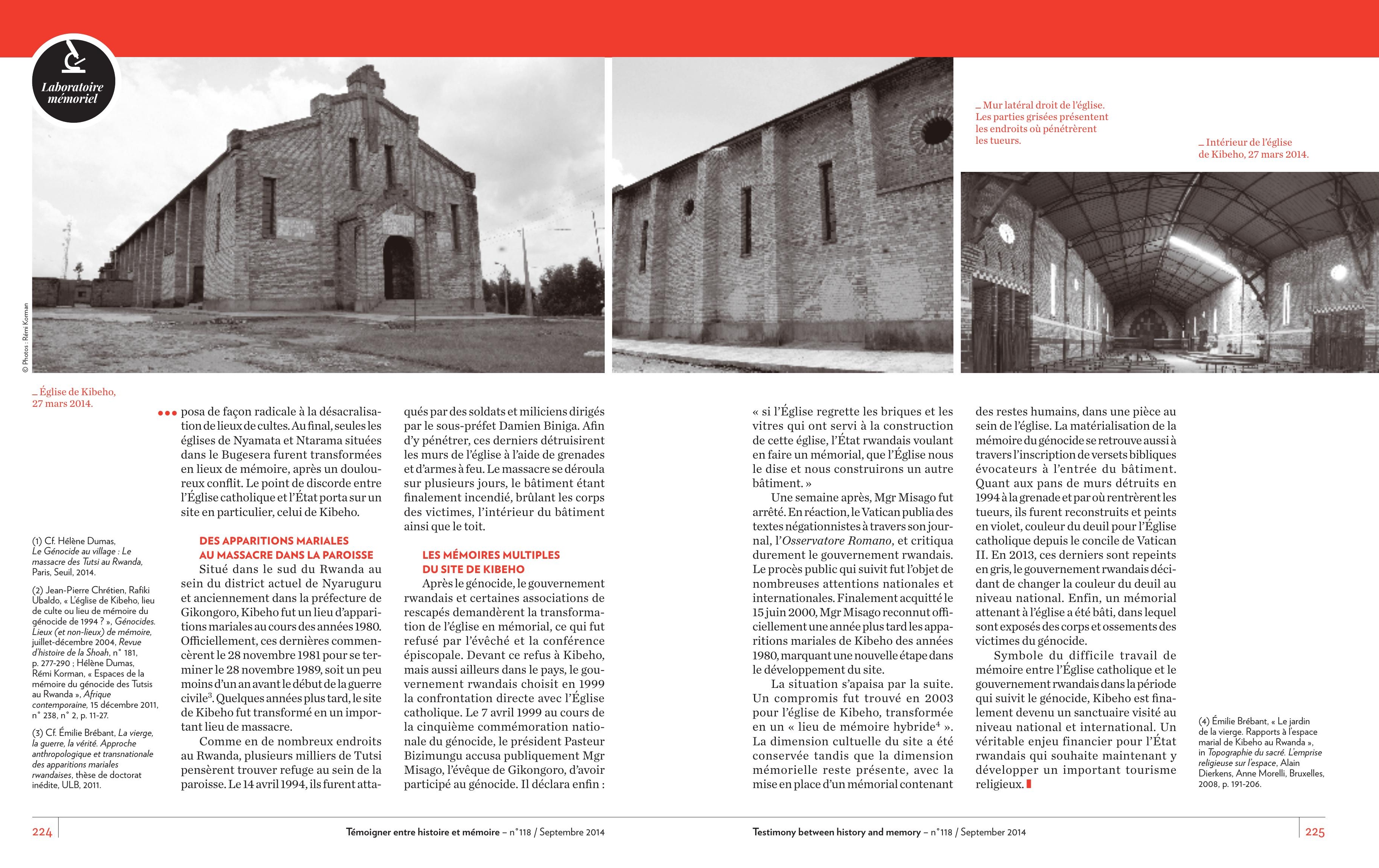




\section{DANS LE PROCHAIN NUMÉRO Laboratoire mémoriel Belgique-België}

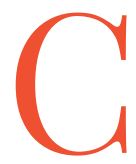
ette rubrique abordera les questions mémorielles touchant à la Belgique par des contributions interdisciplinaires de chercheurs belges et étrangers. Interviews, comptes-rendus de recherches, de livres, annonces de spectacles et d'expositions, dialogues et débats ponctueront ces pages. Il s'agit de suivre de près l'actualité, notamment celle des commémorations du centenaire de la Première Guerre mondiale qui a eu un impact mémoriel important sur les identités nationales et régionales belges.

La rédaction et la coordination de ces pages sont assurées par une équipe de cinq chercheurs flamands et francophones. Issus de différentes disciplines des sciences humaines et sociales, ces chercheurs collaborent étroitement depuis plusieurs années autour des questions de mémoires collectives et des identités belges.

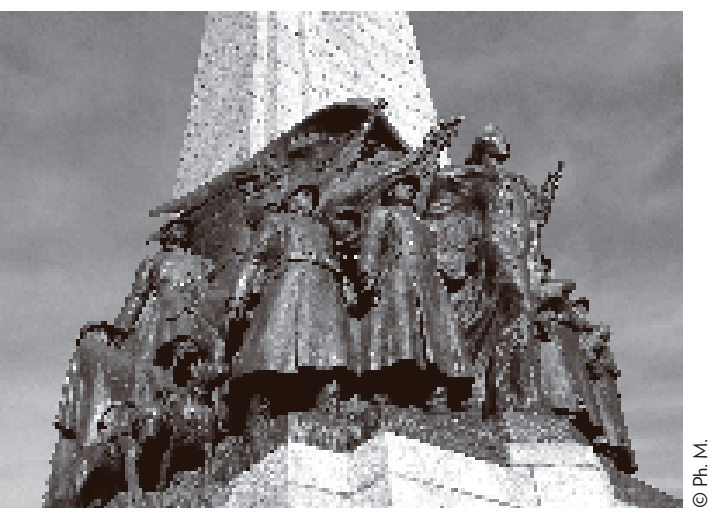

Les diverses représentations du passé constituent le cœur des recherches menées par Marnix Beyen, qui s'intéresse à l'histoire de la Belgique et des Pays-Bas aux XIX et $X X^{e}$ siècles. Il étudie en particulier la construction d'identités politiques à travers des master narratives sur le passé et des pratiques ou objets commémoratifs (tels que le roman historique, les commémorations, les noms de rues) donnant une forme concrète à ces récits.

Elke Brems est coordinatrice du Centre for Reception Studies (Centre d'études de réception) de la KULeuven à Bruxelles. Sa recherche est située à l'intersection de la théorie littéraire et de la traductologie. Plus précisément, elle se concentre sur les différents aspects du transfert culturel - sur la circulation de la littérature et de la culture par la réception, la traduction et l'adaptation de textes. En général, ses analyses portent sur la littérature néerlandaise de la première moitié du XXe siècle et son rapport avec des littératures étrangères.

Olivier Luminet est professeur de psychologie (UCL et ULB) et Directeur de recherche au Fonds de la recherche scientifique (FRS-FNRS). Ses recherches portent notamment sur les liens entre émotions, identité et souvenir individuels et collectifs. Récemment, il a envisagé ces questions en lien avec I'histoire de Belgique (affaire de Louvain, souvenirs intergénérationnels liés à la Seconde Guerre mondiale, sentiments d'honneur et de honte parmi les soldats de la Première Guerre mondiale). Il a notamment coordonné l'ouvrage BelgiqueBelgië. Un état, deux mémoires collectives? (2012, publié en néerlandais sous le titre België-Belgique. Een staat, twee collectieve geheugens?).

Laurence van Ypersele est professeur à l'UCL où elle enseigne l'histoire contemporaine. Elle est l'auteur ou la coauteure de plusieurs ouvrages sur la Première Guerre mondiale, dont De la guerre de l'ombre aux ombres de la guerre (Labor, 2004), Le roi Albert, histoire d'un mythe (Labor, 2006), La Patrie crie vengeance (Le Cri, 2008), Je serai fusillé demain (Racine, 2011) et Bruxelles, la mémoire et la guerre (La Renaissance du Livre, 2014).

Geneviève Warland (UCL), historienne et philosophe, spécialisée en historiographie et en théorie de l'histoire (XIXe-XXe siècles). Elle étudie plus particulièrement les usages publics de l'histoire dans la formation des identités nationales (Allemagne, Belgique, France et Pays-Bas) ainsi que les réseaux d'historiens dans une perspective transnationale. L'activité d'historien-témoin est une thématique qui fait l'objet de ses recherches actuelles concernant la Première Guerre mondiale. 